\title{
$S$-hypersimplices, pulling triangulations, and monotone paths
}

\author{
Sebastian Manecke Raman Sanyal Jeonghoon So \\ Institut für Mathematik \\ Goethe-Universität Frankfurt \\ Germany \\ manecke@math.uni-frankfurt.de \\ sanyal@math. uni-frankfurt.de \\ jeonghoon.so@stud.uni-frankfurt.de
}

Submitted: Jan 17, 2019; Accepted: May 28, 2020; Published: Jul 24, 2020

(C) The authors. Released under the CC BY-ND license (International 4.0).

\begin{abstract}
An $S$-hypersimplex for $S \subseteq\{0,1, \ldots, d\}$ is the convex hull of all $0 / 1$-vectors of length $d$ with coordinate sum in $S$. These polytopes generalize the classical hypersimplices as well as cubes, crosspolytopes, and halfcubes. In this paper we study faces and dissections of $S$-hypersimplices. Moreover, we show that monotone path polytopes of $S$-hypersimplices yield all types of multipermutahedra. In analogy to cubes, we also show that the number of simplices in a pulling triangulation of a halfcube is independent of the pulling order.
\end{abstract}

Mathematics Subject Classifications: 52B20, 52B12

\section{Introduction}

The cube $\square_{d}=[0,1]^{d}$ together with the simplex $\Delta_{d}=\operatorname{conv}\left(\mathbf{0}, \mathbf{e}_{1}, \ldots, \mathbf{e}_{d}\right)$ and the cross-polytope $\diamond_{d}=\operatorname{conv}\left( \pm \mathbf{e}_{1}, \ldots, \pm \mathbf{e}_{d}\right)$ constitute the Big Three, three infinite families of convex polytopes whose geometric and combinatorial features make them ubiquitous throughout mathematics. A close cousin to the cube is the (even) halfcube

$$
H_{d}:=\operatorname{conv}\left(\mathbf{p} \in\{0,1\}^{d}: p_{1}+\cdots+p_{d} \text { even }\right) .
$$

The halfcubes $H_{1}$ and $H_{2}$ are a point and a segment, respectively, but for $d \geqslant 3, H_{d} \subset \mathbb{R}^{d}$ is a full-dimensional polytope. The 5 -dimensional halfcube was already described by Thomas Gosset [11] in his classification of semi-regular polytopes. In contemporary mathematics, halfcubes appear under the name of demi(hyper)cubes [7] or parity polytopes [26]. In particular the name 'parity polytope' suggests a connection to combinatorial optimization 
and polyhedral combinatorics; see $[6,10]$ for more. However, halfcubes also occur in algebraic/topological combinatorics $[13,14]$, convex algebraic geometry [22], and in many more areas.

In this paper, we investigate basic properties of the following class of polytopes that contains cubes, simplices, cross-polytopes, and halfcubes. For a nonempty subset $S$ of $[0, d]:=\{0,1, \ldots, d\}$, we define the $\boldsymbol{S}$-hypersimplex

$$
\Delta(d, S):=\operatorname{conv}\left(\mathbf{v} \in\{0,1\}^{d}: v_{1}+v_{2}+\cdots+v_{d} \in S\right) .
$$

In the context of combinatorial optimization these polytopes were studied by Grötschel [15] associated to cardinality homogeneous set systems. Our name and notation derive from the fact that if $S=\{k\}$ is a singleton, then $\Delta(d, S)=: \Delta(d, k)$ is the well-known $(\boldsymbol{d}, \boldsymbol{k})$-hypersimplex, the convex hull of all vectors $\mathbf{v} \in\{0,1\}^{d}$ with exactly $k$ entries equal to 1 . This is a $(d-1)$-dimensional polytope for $0<k<d$ that makes prominent appearances in combinatorial optimization as well as in algebraic geometry [19]. We call $S$ proper, if $\Delta(d, S)$ is a $d$-dimensional polytope, which, for $d>1$, is precisely the case if $|S| \neq 1$ and $S \neq\{0, d\}$. For appropriate choices of $S \subseteq[0, d]$, we get

- the cube $\square_{d}=\Delta(d,[0, d])$,

- the even halfcube $H_{d}=\Delta(d,[0, d] \cap 2 \mathbb{Z})$,

- the simplex $\Delta_{d}=\Delta(d,\{0,1\})$, and

- the cross-polytope $\Delta(d,\{1, d-1\})$ (up to linear isomorphism).

In Section 2, we study the vertices, edges, and facets of $S$-hypersimplices.

Our study is guided by a nice decomposition of $S$-hypersimplices into Cayley polytopes of hypersimplices.

In Section 3 we return to the halfcube. A combinatorial $d$-cube has the interesting property that all pulling triangulations have the same number of $d$-dimensional simplices. The Freudenthal or staircase triangulation is a pulling triangulation and shows that the number of simplices is exactly $d$ !. We show that the number of simplices in any pulling triangulation of $H_{d}$ is independent of the order in which the vertices are pulled. Moreover, we relate the full-dimensional simplices in any pulling triangulation of $H_{d}$ to partial permutations and show that their number is given by

$$
t(d)=\sum_{l=3}^{d} \frac{d !}{l !}\left(2^{l-1}-l\right) .
$$

For a polytope $P \subset \mathbb{R}^{d}$ and a linear function $\ell: \mathbb{R}^{d} \rightarrow \mathbb{R}$, Billera and Sturmfels [4] associate the monotone path polytope $\Sigma_{\ell}(P)$. This is a $(\operatorname{dim} P-1)$-dimensional polytope whose vertices parametrize all coherent $\ell$-monotone paths of $P$. As a particularly nice example, they show in [4, Example 5.4] that the monotone path polytope $\Sigma_{c}\left(\square_{d}\right)$, where $\mathrm{c}$ is the linear function $\mathrm{c}(\mathbf{x})=x_{1}+x_{2}+\cdots+x_{d}$, is, up to homothety, the polytope

$$
\Pi_{d-1}=\operatorname{conv}((\sigma(1), \ldots, \sigma(d)): \sigma \text { permutation of }[d]) .
$$

For a point $\mathbf{p} \in \mathbb{R}^{d}$, the convex hull of all permutations of $\mathbf{p}$ is called the permutahedron $\Pi(\mathbf{p})$ and we refer to $\Pi_{d-1}=\Pi(1,2, \ldots, d)$ as the standard permutahedron. If 
$\mathbf{p}$ has $d$ distinct coordinates, then $\Pi(\mathbf{p})$ is combinatorially (even normally) equivalent to $\Pi_{d-1}$. For the case that $\mathbf{p}$ has repeated entries, these polytopes were studied by BilleraSarangarajan [3] under the name of multipermutahedra. In Section 4, we study maximal c-monotone paths in the vertex-edge-graph of $\Delta(d, S)$. We show that all c-monotone paths of $\Delta(d, S)$ are coherent and that essentially all multipermutahedra $\Pi(\mathbf{p})$ for $\mathbf{p} \in[0, d-1]^{d}$ occur as monotone path polytopes of $S$-hypersimplices.

We close with some questions and ideas regarding $S$-hypersimplices in Section 5.

\section{$2 \quad S$-hypersimplices}

The vertices of the $d$-cube can be identified with sets $A \subseteq[d]$ and we write $\mathbf{e}_{A} \in\{0,1\}^{d}$ for the point with $\left(\mathbf{e}_{A}\right)_{i}=1$ if and only if $i \in A$. Let $S \subseteq[0, d]$. Since $\Delta(d, S)$ is a vertex-induced subpolytope of the cube, it is immediate that the vertices of $\Delta(d, S)$ are in bijection to

$$
\left(\begin{array}{c}
{[d]} \\
S
\end{array}\right):=\{A \subseteq[d]:|A| \in S\}
$$

This gives the number of vertices as $|V(\Delta(d, S))|=\sum_{s \in S}\left(\begin{array}{l}d \\ s\end{array}\right)$.

For a polytope $P \subset \mathbb{R}^{d}$ and a vector $\mathbf{c} \in \mathbb{R}^{d}$, let

$$
P^{\mathbf{c}}:=\{\mathbf{x} \in P:\langle\mathbf{c}, \mathbf{x}\rangle \geqslant\langle\mathbf{c}, \mathbf{y}\rangle \text { for all } \mathbf{y} \in P\}
$$

be the face in direction c. For example, unless $S=\{0\}, \Delta(d, S)^{\mathbf{e}_{i}}$ is the convex hull of all $\mathbf{e}_{A}$ with $A \in\left(\begin{array}{c}{[d]} \\ S\end{array}\right)$ with $i \in A$. Likewise, unless $S=\{d\}, \Delta(d, S)^{-\mathbf{e}_{i}}=\operatorname{conv}\left(\mathbf{e}_{A}\right.$ : $\left.A \in\left(\begin{array}{c}{[d]} \\ S\end{array}\right), i \notin A\right)$. Under the identification $\left\{\mathbf{x}: x_{i}=1\right\} \cong \mathbb{R}^{d-1}$, this gives for $|S|>1$

$$
\begin{aligned}
\Delta(d, S)^{\mathbf{e}_{i}} \cong \Delta\left(d-1, S^{+}\right) & \text {where } S^{+}:=\{s-1: s \in S, s>0\} \\
\Delta(d, S)^{-\mathbf{e}_{i}} \cong \Delta\left(d-1, S^{-}\right) & \text {where } S^{-}:=\{s: s \in S, s<d-1\} .
\end{aligned}
$$

These faces will be helpful in determining the edges of $\Delta(d, S)$. For two sets $A, B \subseteq[d]$, we denote the symmetric difference of $A$ and $B$ by $A \triangle B:=(A \cup B) \backslash(A \cap B)$. For two points $\mathbf{p}, \mathbf{q} \in \mathbb{R}^{d}$, we write $[\mathbf{p}, \mathbf{q}]$ for the segment joining $\mathbf{p}$ to $\mathbf{q}$.

Theorem 1. Let $S=\left\{0 \leqslant s_{1}<\cdots<s_{k} \leqslant d\right\}$ and $A, B \in\left(\begin{array}{c}{[d]} \\ S\end{array}\right)$ with $|A|=s_{i} \leqslant s_{j}=|B|$. Then $\left[\mathbf{e}_{A}, \mathbf{e}_{B}\right]$ is an edge of $\Delta(d, S)$ if and only if

(i) $A \subset B$ and $j=i+1$, or

(ii) $i=j,|A \triangle B|=2$, and $\left\{s_{i}-1, s_{i}+1\right\} \not \subset S$.

Proof. Let $A, B \in\left(\begin{array}{c}{[d]} \\ S\end{array}\right)$. If $i \in A \cap B$, then $\left[\mathbf{e}_{A}, \mathbf{e}_{B}\right]$ is an edge of $\Delta(d, S)$ if and only if $\left[\mathbf{e}_{A}, \mathbf{e}_{B}\right]$ is an edge of $\Delta(d, S)^{\mathbf{e}_{i}}$. By $(1), \Delta(d, S)^{\mathbf{e}_{i}} \cong \Delta\left(d-1, S^{+}\right)$and $\left[\mathbf{e}_{A}, \mathbf{e}_{B}\right] \cong\left[\mathbf{e}_{A \backslash i}, \mathbf{e}_{B \backslash i}\right]$. Hence we can assume $A \cap B=\varnothing$. For $i \in[d] \backslash(A \cup B)$, we consider $\Delta(d, S)^{-\mathbf{e}_{i}}$ and by the same argument we may also assume that $A \cup B=[d]$.

If $A=\varnothing$, then $B=[d]$ and $\left[\mathbf{e}_{A}, \mathbf{e}_{B}\right]$ meets every $\Delta(d, k)$ in the relative interior for $0<k<d$. Hence $\left[\mathbf{e}_{A}, \mathbf{e}_{B}\right]$ is an edge if and only if $S=\{0, d\}$, which gives us (i). 
If $0<s_{i}=|A|$, then let $i \in A$ and $j \in B$. Then $\left[\mathbf{e}_{A}, \mathbf{e}_{B}\right]$ and $\left[\mathbf{e}_{A^{\prime}}, \mathbf{e}_{B^{\prime}}\right]$ have the same midpoint for $A^{\prime}=(A \backslash i) \cup j$ and $B^{\prime}=(B \backslash j) \cup i$. Thus $\left[\mathbf{e}_{A}, \mathbf{e}_{B}\right]$ is an edge of $\Delta(d, S)$ if and only if $\left(A^{\prime}, B^{\prime}\right)=(B, A)$. This is the case precisely when $|A \triangle B|=2$ and $A \cap B, A \cup B \notin\left(\begin{array}{c}{[d]} \\ S\end{array}\right)$.

Theorem 1 makes the number of edges readily available.

Corollary 2. The number of edges of $\Delta(d, S)$ is

$$
\sum_{i=1}^{k}\left(\begin{array}{c}
d-s_{i} \\
s_{i+1}-s_{i}
\end{array}\right)\left(\begin{array}{l}
d \\
s_{i}
\end{array}\right)+\sum_{j} \frac{s_{j}\left(d-s_{j}\right)}{2}\left(\begin{array}{c}
d \\
s_{j}
\end{array}\right),
$$

where $s_{k+1}:=0$ and the second sum is over all $1 \leqslant j \leqslant k$, such that $\left\{s_{j}-1, s_{j}+1\right\} \not \subset S$.

Let us illustrate Theorem 1 for the classical examples of $S$-hypersimplices. For the cube $\square_{d}=\Delta(d,[0, d])$ it states, that the edges are of the form $\left[\mathbf{e}_{A}, \mathbf{e}_{B}\right]$ for any $A \subset B \subseteq[d]$ such that $|A|+1=|B|$. For the halfcube $H_{d}=\Delta(d,[0, d] \cap 2 \mathbb{Z})$ we infer that there are $d(d-1) 2^{d-3}$ many edges for $d \geqslant 3$. As for the cross-polytope $\Delta(d,\{1, d-1\})$, every two vertices are connected by an edge, except for $\mathbf{e}_{\{i\}}$ and $\mathbf{e}_{[d] \backslash\{i\}}$ for all $i \in[d]$.

Theorem 1 states that there are no long edges of $\Delta(d, S)$. We can make use of this fact to get a canonical decomposition of $\Delta(d, S)$. For $\lambda \in \mathbb{R}$, define the hyperplane

$$
H(\lambda):=\left\{\mathbf{x} \in \mathbb{R}^{d}: x_{1}+\cdots+x_{d}=\lambda\right\} .
$$

We note the following consequence of Theorem 1 .

Corollary 3. Let $S \subseteq[0, d]$ and $s \in S$. Then $\Delta(d, S) \cap H(s)=\Delta(d, s)$.

Proof. Every vertex $\mathbf{v}$ of $\Delta(d, S) \cap H(s)$ is of the form $F \cap H(s)$ for a unique inclusionminimal face $F \subseteq \Delta(d, S)$ of dimension $\leqslant 1$. If $F$ is an edge, then its endpoints $\mathbf{e}_{A}, \mathbf{e}_{B}$ satisfy $|A|<s<|B|$ which contradicts Theorem 1 . Hence $F=\mathbf{e}_{C}$ for some $C \subseteq[d]$ with $|C|=s$.

If $S=\left\{s_{1}<\cdots<s_{k}\right\}$ with $k \geqslant 2$, then we can decompose

$$
\Delta(d, S)=\Delta\left(d, s_{1}, s_{2}\right) \cup \Delta\left(d, s_{2}, s_{3}\right) \cup \cdots \cup \Delta\left(d, s_{k-1}, s_{k}\right),
$$

where we set $\Delta(d, k, l):=\Delta(d,\{k, l\})=\operatorname{conv}(\Delta(d, k) \cup \Delta(d, l))$ for $0 \leqslant k<l \leqslant d$. The polytope $\Delta(d, k, l)$ is the Cayley polytope of $\Delta(d, k)$ and $\Delta(d, l)$. Moreover, for $i<j$, we see that $\Delta\left(d, s_{i}, s_{i+1}\right) \cap \Delta\left(d, s_{j}, s_{j+1}\right)=\Delta\left(d, s_{j}\right)$ if $j=i+1$ and $=\varnothing$ otherwise.

Before we determine the facets of $\Delta(d, S)$, we recall some properties of permutahedra from [3] that we will also need in Section 4. Let us say that a point $\mathbf{p} \in \mathbb{R}^{d}$ is decreasing if $p_{1} \geqslant p_{2} \geqslant \cdots \geqslant p_{d}$. The permutahedron associated to $\mathbf{p}$ is the polytope

$$
\Pi(\mathbf{p}):=\operatorname{conv}\left(\sigma \mathbf{p}:=\left(p_{\sigma(1)}, p_{\sigma(2)}, \ldots, p_{\sigma(d)}\right): \sigma \text { permutation of }[d]\right) .
$$

Unless $p_{i}=p_{j}$ for all $i \neq j, \Pi(\mathbf{p})$ is a polytope of dimension $d-1$ with affine hull given by $H\left(p_{1}+\cdots+p_{d}\right)$.

Notice that $\Pi(\mathbf{p})^{\sigma \mathbf{u}}=\sigma^{-1} \Pi(\mathbf{p})^{\mathbf{u}}$. Thus, if we want to determine the face $\Pi(\mathbf{p})^{\mathbf{u}}$ up to permutation of coordinates, we can assume that $\mathbf{u}$ is decreasing. The Minkowski sum of two polytopes $P, Q \subset \mathbb{R}^{d}$ is the polytope $P+Q=\{\mathbf{p}+\mathbf{q}: p \in P, q \in Q\}$. 
Proposition 4. Let $\mathbf{p}, \mathbf{q} \in \mathbb{R}^{d}$ be decreasing. Then

$$
\Pi(\mathbf{p})+\Pi(\mathbf{q})=\Pi(\mathbf{p}+\mathbf{q}) .
$$

Proof. Set $P:=\Pi(\mathbf{p})+\Pi(\mathbf{q})$. Clearly $\sigma(\mathbf{p}+\mathbf{q})=\sigma \mathbf{p}+\sigma \mathbf{q}$ for all permutations $\sigma$ and therefore every vertex of $\Pi(\mathbf{p}+\mathbf{q})$ is a vertex of $P$. For the converse, let $\mathbf{c}$ be such that $P^{\mathbf{c}}=\{\mathbf{v}\}$ is a vertex. Since $P$ is invariant under coordinate permutations, we can assume that $\mathbf{c}$ is decreasing. Furthermore $(\Pi(\mathbf{p})+\Pi(\mathbf{q}))^{\mathbf{c}}=\Pi(\mathbf{p})^{\mathbf{c}}+\Pi(\mathbf{q})^{\mathbf{c}}$ and it follows that $\mathbf{v}=\mathbf{p}+\mathbf{q}$. Hence, every vertex of $P$ is of the form $\sigma(\mathbf{p}+\mathbf{q})$ for some permutation $\sigma$, which completes the proof.

For $\nu_{1}>\nu_{2}>\cdots>\nu_{r}$ and $k_{1}, k_{2}, \ldots, k_{r} \in \mathbb{Z}_{>0}$ such that $k_{1}+\cdots+k_{r}=d$, we set

$$
\left(\nu_{1}^{k_{1}}, \nu_{2}^{k_{2}}, \ldots, \nu_{r}^{k_{r}}\right):=(\underbrace{\nu_{1}, \ldots, \nu_{1}}_{k_{1}}, \underbrace{\nu_{2}, \ldots, \nu_{2}}_{k_{2}}, \ldots, \underbrace{\nu_{r}, \ldots, \nu_{r}}_{k_{r}}) .
$$

For example, the $(d, k)$-hypersimplex is the permutahedron $\Delta(d, k)=\Pi\left(1^{k}, 0^{d-k}\right)$.

The facets of permutahedra were described by Billera-Sarangarajan [3]. We recall their characterization. We write $I^{c}:=[d] \backslash I$ for the complement of $I \subseteq[d]$.

Theorem 5 ([3, Theorem 3.2]). Let $P=\Pi\left(\nu_{1}^{k_{1}}, \ldots, \nu_{r}^{k_{r}}\right)$ and $\mathbf{c} \in \mathbb{R}^{d}$. Then $P^{\mathbf{c}}$ is a facet if and only if $\mathbf{c}=\alpha \mathbf{e}_{I}+\beta \mathbf{e}_{I^{\mathbf{c}}}$ for some $\alpha>\beta$ and $\varnothing \neq I \subset[d]$ and $h=|I|$ satisfies

(a) $k_{1}+1 \leqslant h \leqslant d-k_{r}-1$, or

(b) $h=1$ if $k_{r}<d-1$, or

(c) $h=d-1$ if $k_{1}<d-1$.

The theorem shows, for example, that $\Delta(d, k)$ for $1<k<d-1$ has $2 d$ facets with normals given by $\pm \mathbf{e}_{1}, \ldots, \pm \mathbf{e}_{d}$.

In order to determine the facets of $\Delta(d, S)$, we appeal to the decomposition (2). Let $S=\left\{0 \leqslant s_{1}<s_{2}<\cdots<s_{k} \leqslant d\right\}$ be proper so that $\Delta(d, S) \subset \mathbb{R}^{d}$ is full-dimensional. We write $\mathbf{1}:=\mathbf{e}_{[d]}$ for the all-ones vector. If $s_{1}>0$, then $\Delta\left(d, s_{1}\right)=\Delta(d, S)^{-\mathbf{1}}$ is a facet. Likewise, if $s_{d}<d$, then $\Delta\left(d, s_{k}\right)=\Delta(d, S)^{\mathbf{1}}$ is a facet. If $F \subset \Delta(d, S)$ is any other facet, then its vertices cannot have all the same cardinality. If $s_{i} \in S$ is the minimal cardinality of a vertex in $F$, then $F \cap \Delta\left(d, s_{i}, s_{i+1}\right)$ is a facet of $\Delta\left(d, s_{i}, s_{i+1}\right)$. Hence, as a first step, we determine the facets of $\Delta\left(d, s_{i}, s_{i+1}\right)$ that are not equal to $\Delta\left(d, s_{i}\right)$ and $\Delta\left(d, s_{i+1}\right)$.

Let $S=\{k<l\}$ be proper. An easy calculation shows that

$$
\Delta(d, k, l) \cap H\left(\frac{k+l}{2}\right)=\frac{1}{2}(\Delta(d, k)+\Delta(d, l)) .
$$

Moreover, if $F \subset \Delta(d, k, l)$ is a facet, then $F \cap H\left(\frac{k+l}{2}\right)$ is a facet of the right-hand side and every facet arises that way. Hence it suffices to determine the facets of $\bar{\Delta}(d, k, l):=$ $\Delta(d, k)+\Delta(d, l)$. We will need the notion of a join of two polytopes: If $P, Q \subset \mathbb{R}^{d}$ are polytopes such that their affine hulls are skew, i.e., non-parallel and disjoint, then $P * Q:=\operatorname{conv}(P \cup Q)$ is called the join of $P$ and $Q$. Every $k$-dimensional face of $P * Q$ is of the form $F * G$ where $F \subseteq P$ and $G \subseteq G$ are (possibly empty) faces with $\operatorname{dim} F+\operatorname{dim} G=k-1$. 
Proposition 6. Let $1 \leqslant k<l<d$. In addition to the facets $\Delta(d, k, l)^{\mathbf{1}}=\Delta(d, l)$ and $\Delta(d, k, l)^{-\mathbf{1}}=\Delta(d, k)$, there are

$$
\Delta(d, k, l)^{\mathbf{e}_{i}} \cong \Delta(d-1, k-1, l-1) \quad \text { and } \quad \Delta(d, k, l)^{-\mathbf{e}_{i}} \cong \Delta(d-1, k, l)
$$

for $i=1, \ldots, d$. Every other facet is of the form

$$
\Delta(d, k, l)^{\mathbf{c}} \cong \Delta(h, k) * \Delta(d-h, l-h)
$$

where $\mathbf{c}=(l-h) \mathbf{e}_{I}-(h-k) \mathbf{e}_{I^{c}}$ for any $\varnothing \neq I \subset[d]$ with $k<h:=|I|<l$.

Proof. We first determine the facets of $\bar{\Delta}(d, k, l)$. Using Proposition 4 , we see that $\bar{\Delta}(d, k, l)$ is the permutahedron $\Pi\left(2^{k}, 1^{l-k}, 0^{d-l}\right)$. Theorem 5 yields that the facet directions of $\bar{\Delta}(d, k, l)$ are given $\mathbf{c}=\alpha \mathbf{e}_{I}+\beta \mathbf{e}_{I^{c}}$ for $\varnothing \neq I \subset[d]$ with $|I|=1,|I|=d-1$, or $k<|I|<l$ and $\alpha>\beta$. In particular, for every $I$ there is, up to scaling, a unique choice for $\alpha$ and $\beta$ so that $\Delta(d, k, l)^{\mathbf{c}}$ is a facet.

For $I=\{i\}$ we already observed that $\mathbf{c}=\mathbf{e}_{I}=\mathbf{e}_{i}$ yields a facet linearly isomorphic to $\Delta(d-1, k-1, l-1)$. Likewise, for $[d] \backslash I=\{j\}$, we obtain for $\mathbf{c}=\mathbf{e}_{I}-\mathbf{1}=-\mathbf{e}_{j}$ a facet that is linearly isomorphic to $\Delta(d-1, k, l)$.

For $I \subseteq[d]$ with $k<|I|<l$, we observe that $\mathbf{e}_{A} \in \Delta(d, k)^{\mathbf{e}_{I}}$ if and only if $A \subset I$ and $\mathbf{e}_{A} \in \Delta(d, l)^{\mathbf{e}_{I}}$ if and only if $I \subset A$. Set $h:=|I|$ and $\mathbf{c}=(l-h) \mathbf{e}_{I}-(h-k) \mathbf{e}_{I^{c}}$. For $A \in\left(\begin{array}{c}{[d]} \\ k\end{array}\right)$ we compute

$$
\left\langle\mathbf{c}, \mathbf{e}_{A}\right\rangle=(l-h)|A \cap I|-(h-k)\left|A \cap I^{c}\right| \leqslant(l-h) k
$$

with equality if and only if $A \subset I$. For $A \in\left(\begin{array}{c}{[d]} \\ l\end{array}\right)$, we compute

$$
\left\langle\mathbf{c}, \mathbf{e}_{A}\right\rangle=(l-h)|A \cap I|-(h-k)\left|A \cap I^{c}\right| \leqslant(l-h) h-(h-k)(l-h)=(l-h) k
$$

with equality if and only if $I \subset A$. Hence the hyperplane $H=\{\mathbf{x}:\langle\mathbf{c}, \mathbf{x}\rangle=(l-h) k\}$ supports $\Delta(d, k, l)$ in a facet, since $H$ also supports a facet of $\bar{\Delta}(d, k, l)$. In particular, $\Delta(d, k) \cap H \cong \Delta(h, k)$ under the identification $\left\{\mathbf{x}: x_{i}=0\right.$ for $\left.i \notin I\right\} \cong \mathbb{R}^{h}$. Likewise $\Delta(d, l) \cap H \cong \Delta(d-h, l-h)$ under the identification $\left\{\mathbf{x}: x_{i}=1\right.$ for $\left.i \in I\right\} \cong \mathbb{R}^{d-h}$. This also shows that the given subspaces are skew and, since they lie in $H(k)$ and $H(l)$ respectively, are disjoint. This shows that $\Delta(d, l, k) \cong \Delta(h, k) * \Delta(d-h, l-h)$.

It follows from Proposition 6 that $\Delta(d, k, l)$ and $\Delta(d, l, m)$ for $0<k<l<m<d$ never have facet normals of type (v) in common. This gives us the following description of facets of $S$-hypersimplices; see also [15].

Theorem 7. Let $S=\left\{0 \leqslant s_{1}<\cdots<s_{k} \leqslant d\right\}$ be proper. Then $\Delta(d, S)$ has the following facets

(i) $\Delta(d, S)^{\mathbf{1}}=\Delta\left(d, s_{k}\right)$ provided $s_{k}<d$;

(ii) $\Delta(d, S)^{-\mathbf{1}}=\Delta\left(d, s_{1}\right)$ provided $0<s_{1}$;

(iii) $\Delta(d, S)^{\mathbf{e}_{i}} \cong \Delta\left(d-1, S^{+}\right)$for $i=1, \ldots, d$ provided $S^{+}$is proper;

(iv) $\Delta(d, S)^{-\mathbf{e}_{i}} \cong \Delta\left(d-1, S^{-}\right)$for $i=1, \ldots, d$ provided $S^{-}$is proper; 
(v) $\Delta(d, S)^{\mathbf{u}_{I}} \cong \Delta\left(h, h-s_{i}\right) * \Delta\left(d-h, s_{i+1}-h\right)$ where $I \subset[d]$ with $s_{i}<|I|=: h<s_{i+1}$ for some $0<i<k$ and $\mathbf{u}_{I}:=\left(s_{i+1}-h\right) \mathbf{e}_{I}-\left(h-s_{i}\right) \mathbf{e}_{I^{c}}$.

Proof. By decomposition (2), every facet $F$ of $\Delta(d, S)$ determines a facet of $\Delta\left(d, s_{i}, s_{i+1}\right)$ for some $1 \leqslant i<k$ and $F$ is decomposed by this collection of facets. By examining the possible facet normals of $\Delta\left(d, s_{i}, s_{i+1}\right)$, the statement readily follows.

If $S=[0, d]$, then Theorem 7 gives us that $\square_{d}$ has exactly $2 d$ facets in the coordinate directions $\pm \mathbf{e}_{i}$ for $i=1, \ldots, d$. The facets are again cubes as $[0, d]^{ \pm}=[0, d-1]$. The $d$-dimensional crosspolytope $\nabla_{d} \cong \Delta(d,\{1, d-1\})$ has $2^{d}$ facets. The two facets of type (i), (ii), and those of type (iii) and (iv) are simplices. As for type (v) this is a join of two simplices and thus also a simplex.

The description of combinatorial type of each facet also leads to the number of $k$ dimensional faces for $0 \leqslant k<d$; cf. [21].

\section{Pulling triangulations}

A subdivision $\mathcal{S}$ of a $d$-dimensional polytope $P \subset \mathbb{R}^{d}$ is a collection $\mathcal{S}=\left\{P_{1}, \ldots, P_{m}\right\}$ of $d$-polytopes such that $P=P_{1} \cup \cdots \cup P_{m}$ and $P_{i} \cap P_{j}$ is a face of $P_{i}$ and $P_{j}$ for all $1 \leqslant i<j \leqslant m$. If all polytopes $P_{i}$ are simplices, then $\mathcal{S}$ is called a triangulation. Triangulations are the method-of-choice for various computations on polytopes including volume, lattice point counting, or, more generally, computing valuations; see [8].

A powerful method for computing a triangulation is the so-called pulling triangulation. Let $P$ be a $d$-polytope and $\mathbf{v} \in V(P)$ a vertex. Let $F_{1}, \ldots, F_{m}$ be the facets of $P$ not containing $\mathbf{v}$. A key insight is that the collection of polytopes

$$
P_{i}:=\mathbf{v} * F_{i}:=\operatorname{conv}\left(F_{i} \cup\{\mathbf{v}\}\right) \quad \text { for } i=1, \ldots, m
$$

constitutes a subdivision of $P$. This idea can be extended to obtain triangulations. Let $\preceq$ be a partial order on the vertex set $V(P)$ such that every nonempty face $F \subseteq P$ has a unique minimal element with respect to $\preceq$. We denote the minimal vertex of $F$ by $\mathbf{v}_{F}$. The pulling triangulation $\mathrm{Pull}_{\preceq}(P)$ of $P$ is recursively defined as follows. If $P$ is a simplex, then $\operatorname{Pull}_{\preceq}(P)=\{P\}$. Otherwise, we define

$$
\operatorname{Pull}_{\preceq}(P)=\bigcup_{F} \mathbf{v}_{P} * \operatorname{Pull}_{\preceq}(F),
$$

where the union is over all facets $F \subset P$ that do not contain $\mathbf{v}_{P}$ and where $\mathbf{v}_{P} * \operatorname{Pull}_{\preceq}(F):=$ $\left\{\mathbf{v}_{P} * Q: Q \in \operatorname{Pull}_{\preceq}(F)\right\}$.

For the cube $\square_{d}$, or more generally the class of compressed polytopes [25], it can be shown that every simplex $S$ in a pulling triangulation of $\square_{d}$ has the same volume $\frac{1}{d !}$. Thus, every pulling triangulation has exactly $d$ ! many simplices, independent of the chosen order $\preceq$.

Recall that the halfcube is the $S$-hypersimplex $H_{d}=\Delta(d,[0, d] \cap 2 \mathbb{Z})$. For $d \geqslant 5$ it is not true that the simplices in a pulling triangulation of $H_{d}$ all have the same volume. The 
main result of this section is that still the number of simplices in a pulling triangulation is independent of the choice of $\preceq$.

Theorem 8. Every pulling triangulation of $H_{d}$ has the same number of simplices. The number of simplices $t(d):=\left|\mathrm{Pull}_{\preceq}\left(H_{d}\right)\right|$ is given by

$$
t(d)=\sum_{l=3}^{d} \frac{d !}{l !}\left(2^{l-1}-l\right) .
$$

The proof of Theorem 8 is in two parts. We first show that the number of simplices of $\operatorname{Pull}_{\preceq}\left(H_{d}\right)$ is independent of $\preceq$. This yields a recurrence relation on $t(d)$. In the second part we review the construction of $\mathrm{Pull}_{\preceq}\left(H_{d}\right)$ from the perspective of choosing facets, which yields a combinatorial interpretation for $t(d)$ and which then verifies the stated expression.

From Theorem 7 we infer the following description of facets of $H_{d}$ for $d \geqslant 3$ : For every $i=1, \ldots, d$ we have

$$
\begin{aligned}
H_{d}^{-\mathbf{e}_{i}} & =H_{d} \cap\left\{\mathbf{x}: x_{i}=0\right\} \cong H_{d-1}, \\
H_{d}^{\mathbf{e}_{i}} & =H_{d} \cap\left\{\mathbf{x}: x_{i}=1\right\} \cong H_{d-1},
\end{aligned}
$$

where the last isomorphism is realized by reflection in a hyperplane $\left\{\mathbf{x}: x_{j}=\frac{1}{2}\right\}$ for $j \neq i$. The remaining facets of $H_{d}$ are provided by Theorem $7(\mathrm{v})$ and, in case $d$ is odd, by (i): For $B \subseteq[d]$ with $|B|$ odd and $\mathbf{u}_{B}=\mathbf{e}_{B}-\mathbf{e}_{B^{c}}$, we have

$$
H_{d}^{\mathbf{u}_{B}}=H_{d} \cap\left\{\mathbf{x}:\left\langle\mathbf{e}_{B}, \mathbf{x}\right\rangle-\left\langle\mathbf{e}_{B^{c}}, \mathbf{x}\right\rangle=|B|-1\right\} \cong \Delta_{d-1} .
$$

Proposition 9. The number $t(d)$ of simplices in a pulling triangulation of $H_{d}$ satisfies

$$
t(d)=d \cdot t(d-1)+2^{d-1}-d
$$

for $d \geqslant 4$ and $t(d)=1$ for $d \leqslant 3$.

Proof. We prove the result by induction on $d$. For $d=1,2,3$, we note that $H_{d}$ is itself a simplex and thus there is nothing to prove.

For $d \geqslant 4$, let $A \subseteq[d]$ be an even subset such that $\mathbf{e}_{A} \in\{0,1\}^{d}$ is the minimal vertex of $P$ with respect to $\preceq$. By the discussion preceding the proposition, the facets not containing $\mathbf{e}_{A}$ are $H_{d}^{\mathbf{e}_{i}} \cong H_{d-1}$ for $i \notin A, H_{d}^{-\mathbf{e}_{i}} \cong H_{d-1}$ for $i \in A$, and $H_{d}^{\mathbf{u}_{B}} \cong \Delta_{d-1}$ for

$$
B \in \mathcal{B}:=\{B \subseteq[d]:|B| \text { odd, }|A \triangle B|>1\} .
$$

Note that $|\mathcal{B}|=2^{d-1}-d$. Thus it follows from (3) that

$$
\begin{aligned}
t(d) & =\left|\operatorname{Pull}_{\preceq}\left(H_{d}\right)\right|=\sum_{i \in A}\left|\operatorname{Pull}_{\preceq}\left(H_{d}^{-\mathbf{e}_{i}}\right)\right|+\sum_{i \notin A}\left|\operatorname{Pull}_{\preceq}\left(H_{d}^{\mathbf{e}_{i}}\right)\right|+\sum_{B \in \mathcal{B}}\left|\operatorname{Pull}_{\preceq}\left(H_{d}^{\mathbf{u}_{B}}\right)\right| \\
& =d \cdot t(d-1)+2^{d-1}-d,
\end{aligned}
$$

where the last equality follows by induction. 
Let $P \subset \mathbb{R}^{d}$ be a full-dimensional polytope with suitable partial order $\preceq$ on $V(P)$. Every simplex in $\mathrm{Pull}_{\preceq}(P)$ corresponds to a chain of faces

$$
P=G_{0} \supset G_{1} \supset G_{2} \supset \cdots \supset G_{k}
$$

such that $\operatorname{dim} G_{i}=d-i$ and $G_{k}$ is a simplex of dimension $d-k$. The corresponding simplex is then given by $\mathbf{v}_{G_{0}} * \mathbf{v}_{G_{1}} * \cdots * G_{k}$. If $P$ is a simple polytope with facets $F_{1}, \ldots, F_{m}$, then any such chain of faces is given by an ordered sequence of distinct indices $h_{1}, h_{2}, \ldots, h_{k}$ such that

for all $i=0, \ldots, k$.

$$
G_{i}=F_{h_{1}} \cap F_{h_{2}} \cap \cdots \cap F_{h_{i}}
$$

For the $d$-dimensional cube $\square_{d}$, the facets can be described by $(i, \delta) \in[d] \times\{0,1\}$ so that

$$
K_{i}^{\delta}:=\square_{d} \cap\left\{x_{i}=\delta\right\} \cong \square_{d-1} .
$$

The only faces of $\square_{d}$ that are simplices have dimensions $\leqslant 1$ and thus simplices in Pull $_{\preceq}\left(\square_{d}\right)$ correspond to sequences $\left(i_{1}, \delta_{1}\right), \ldots,\left(i_{d-1}, \delta_{d-1}\right) \in[d] \times\{0,1\}$ with $i_{s} \neq i_{t}$ for $s \neq t$. Thus, if we choose $i_{d}$ such that $\left\{i_{1}, \ldots, i_{d-1}, i_{d}\right\}=[d]$, then every simplex of Pull $_{\preceq}\left(\square_{d}\right)$ determines a permutation $\sigma=i_{1} i_{2} \cdots i_{d}$ of $[d]$.

Observe that for any vertex $\mathbf{v} \in \square_{d}$ and $i \in[d]$, we have that $\mathbf{v} \in K_{i}^{0}$ or $\mathbf{v} \in K_{i}^{1}$. This means that for any permutation $\sigma=i_{1} i_{2} \cdots i_{d}$ of $[d]$ there are $\delta_{1}, \delta_{2}, \ldots, \delta_{d-1} \in\{0,1\}$ such that $\left(i_{1}, \delta_{1}\right), \ldots,\left(i_{d-1}, \delta_{d-1}\right)$ come from a simplex in $\operatorname{Pull}_{\preceq}\left(\square_{d}\right)$. This shows that $\left|\operatorname{Pull}_{\preceq}\left(\square_{d}\right)\right|=d$ ! independent of the order $\preceq$.

We call a sequence $\tau=i_{1} i_{2} \ldots i_{k}$ with $i_{1}, \ldots, i_{k} \in[d]$ a partial permutation if $i_{s} \neq i_{t}$ for $s \neq t$. We simply write $[d] \backslash \tau$ for $[d] \backslash\left\{i_{1}, \ldots, i_{k}\right\}$. The following Proposition completes the proof of Theorem 8 .

Proposition 10. For any suitable partial order $\preceq$, the simplices of $\mathrm{Pull}_{\preceq}\left(H_{d}\right)$ for $d \geqslant 3$ are in bijection to pairs $(\tau, B)$ where $\tau$ is a partial permutation of $[d]$ and $B \subseteq[d] \backslash \tau$ is a non-singleton subset of odd cardinality.

Proof. Since $H_{3}$ is a simplex and the only admissible pair $(\tau, B)$ is given by the empty partial permutation and $B=[3]$, we assume $d \geqslant 4$. For $i=1, \ldots, d$ and $\delta \in\{0,1\}$, let

$$
F_{i}^{\delta}:=H_{d} \cap\left\{x_{i}=\delta\right\} \cong H_{d-1}
$$

be the halfcube facets of $H_{d}$. The halfcube $H_{d}$ for $d \geqslant 4$ is not a simple polytope. However, it follows from Theorem 7 that the faces of $H_{d}$ are halfcubes or simplices. If $G \subset H_{d}$ is a face linearly isomorphic to a halfcube of dimension $d-k \geqslant 4$, then $G$ is a simple face in the sense that $G$ is precisely the intersection of $k$ halfcube facets. Every chain of faces (4) corresponds to some $\left(i_{1}, \delta_{1}\right), \ldots,\left(i_{k-1}, \delta_{k-1}\right) \in[d] \times\{0,1\}$ such that $G_{k-1}=F_{i_{1}}^{\delta_{1}} \cap \cdots \cap F_{i_{k-1}}^{\delta_{k-1}}$ is isomorphic to $H_{d-k+1}$ and $G_{k}$ is a simplex facet of $G_{k-1}$ not containing $\mathbf{v}_{G_{k-1}}$. This gives rise to a unique partial permutation $\tau=i_{1} i_{2} \ldots i_{k-1}$. To see that any such partial permutation can arise, we observe that again $V\left(H_{d}\right) \subset F_{i}^{0} \cup F_{i}^{1}$ for all $i=1, \ldots, d$. We can identify $G_{k-1}$ with $H_{d-k+1}$ embedded in $\left\{\mathbf{x}: x_{i_{1}}=\cdots=x_{i_{k-1}}=0\right\}$ and $v_{G_{k-1}}=\mathbf{0}$. Now any simplex facet of $H_{d-k+1}$ corresponds to an odd-cardinality subset $B \subset[d] \backslash \tau$ with $|B| \neq 1$. 


\section{Monotone paths}

Let $P \subset \mathbb{R}^{d}$ be a polytope and $\ell: \mathbb{R}^{d} \rightarrow \mathbb{R}$ a linear function. An $\boldsymbol{\ell}$-monotone path of $P$ is a sequence of vertices $W=\mathbf{v}_{1}, \mathbf{v}_{2}, \ldots, \mathbf{v}_{k}$ such that $\left[\mathbf{v}_{i}, \mathbf{v}_{i+1}\right]$ is an edge of $P$ for $i=1, \ldots, k-1$ and

$$
\min \ell(P)=\ell\left(\mathbf{v}_{1}\right)<\ell\left(\mathbf{v}_{2}\right)<\cdots<\ell\left(\mathbf{v}_{k}\right)=\max \ell(P) .
$$

More generally, a collection of faces $F_{1}, F_{2}, \ldots, F_{k}$ of $P$ is an induced subdivision of the segment $\ell(P)$ if $F_{1}^{-\ell}$ and $F_{k}^{\ell}$ is a face of $P^{-\ell}$ and $P^{\ell}$, respectively, and

$$
F_{i}^{\ell}=F_{i+1}^{-\ell}
$$

for $i=1, \ldots, k-1$. If $\ell$ is generic, that is, if $\ell$ is not constant on edges of $P$, then the minimum/maximum of $\ell$ on every nonempty face $F$ is attained at a unique vertex. In this case $F_{i}^{ \pm \ell}$ is a vertex for all $i$ and an induced subdivision is called a cellular string. An induced subdivision $F_{1}^{\prime}, \ldots, F_{h}^{\prime}$ is a refinement if for every $1 \leqslant i \leqslant k$, there are $1 \leqslant s<t \leqslant h$ such that $F_{s}^{\prime}, \ldots, F_{t}^{\prime}$ is an induced subdivision of $\ell\left(F_{i}\right)$. The collection of all induced subdivisions of $\ell(P)$ is partially ordered by refinement and is called the Baues poset of $(P, \ell)$. The minimal elements in the Baues poset are exactly the $\ell$-monotone paths. Monotone paths are quintessential in the study of simplex-type algorithms in linear programming but they are also studied in topology in connection with iterated loop spaces; see $[2,20]$. For the linear function $\mathrm{c}(x)=x_{1}+\cdots+x_{d}$, Corollary 2 readily yields the c-monotone paths of $\Delta(d, S)$.

Corollary 11. Let $S=\left\{s_{1}<s_{2}<\cdots<s_{k}\right\}$ be proper. The c-monotone paths correspond to sequences $A_{1} \subset A_{2} \subset \cdots \subset A_{k}$ with $\left|A_{i}\right|=s_{i}$ for all $i=1, \ldots, k$.

A $\ell$-monotone path $W$ is coherent if $W$ is a monotone path with respect to the shadow-vertex algorithm; see $[5,17]$. That is, if there is linear function $h_{W}: \mathbb{R}^{d} \rightarrow \mathbb{R}$ such that under the projection $\pi: \mathbb{R}^{d} \rightarrow \mathbb{R}^{2}$ given by $\pi(\mathbf{x})=\left(\ell(\mathbf{x}), h_{W}(\mathbf{x})\right)$, the path $W$ is mapped to one of the two paths in the boundary of the polygon $\pi(P)$. Figure 1 shows that in general coherent paths constitute a proper subset of all $\ell$-monotone paths and it is interesting to determine for which pairs $(P, \ell)$ all $\ell$-monotone paths are coherent; see, for example, the recent paper [9]. The $S$-hypersimplices with the linear function c $(x)$ are examples of this.

Proposition 12. Let $S \subseteq[0, d]$ be proper. Then all c-monotone path of $\Delta(d, S)$ are coherent.

Proof. Let $A_{1} \subset A_{2} \subset \cdots \subset A_{k}$ be a c-monotone path. For the linear function

$$
h(\mathbf{x}):=\left\langle\mathbf{1}_{A_{1}}+\cdots+\mathbf{1}_{A_{k}}, \mathbf{x}\right\rangle
$$

it is easy to see that $h\left(\mathbf{1}_{B}\right)$ with $B \in\left(\begin{array}{c}{[d]} \\ S\end{array}\right)$ is maximal if and only if $B \in\left\{A_{1}, \ldots, A_{k}\right\}$. 


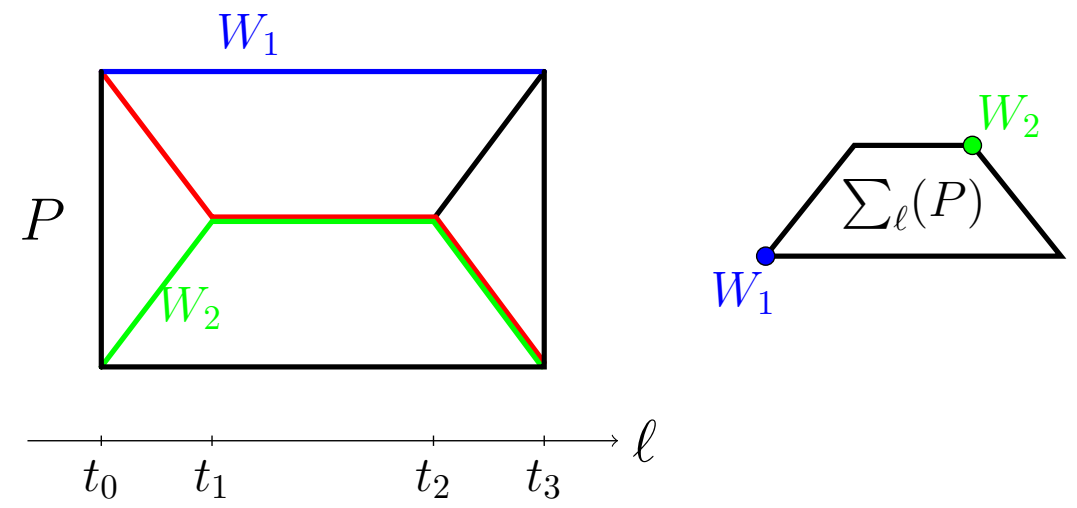

Figure 1: Left: Top view of triangular prism $P$ and linear function $\ell$. Three $\ell$-monotone paths (in red, green, and blue) but the red path is not coherent. Right: Monotone path polytope $\Sigma_{\ell}(P)$.

The monotone path polytope $\Sigma_{\ell}(P)$ is a convex polytope of dimension $\operatorname{dim} P-1$ whose face lattice is isomorphic to the poset of coherent subdivisions. The construction is a special case of fiber polytopes of Billera and Sturmfels [4]. Let $\ell(P)=[a, b] \subset \mathbb{R}$. A section of $(P, \ell)$ is a continuous function $\gamma:[a, b] \rightarrow P$ such that $\ell(\gamma(t))=t$ for all $a \leqslant t \leqslant b$. Following [4], the monotone path polytope is defined as

$$
\Sigma_{\ell}(P)=\operatorname{conv}\left\{\frac{1}{b-a} \int_{P} \gamma d \mathbf{x}: \gamma \text { section }\right\} .
$$

We now determine the monotone path polytopes of $\Delta(d, S)$ with respect to the natural linear function $\mathrm{c}(x)=x_{1}+\cdots+x_{d}$. Let us first observe that for $S \subset[d-1]$ the c-monotone paths of $\Delta(d, S)$ and $\Delta(d, S \cup\{0, d\})$ are in bijection. Clearly every c-monotone path of $\Delta(d, S \cup\{0, d\})$ restricts to a c-monotone path of $\Delta(d, S)$. Conversely, if $A_{1} \subset \cdots \subset A_{k}$ corresponds to a c-monotone path, then $\varnothing=: A_{0} \subset A_{1} \subset \cdots \subset A_{k} \subset A_{k+1}=[d]$ is the unique extension to a c-monotone path of $\Delta(d, S \cup\{0, d\})$.

Theorem 13. Let $S=\left\{0=s_{0} \leqslant s_{1}<s_{2}<\cdots<s_{k-1}<s_{k}=d\right\}$ be proper. Then

$$
\frac{1}{2} \mathbf{1}+d \cdot \Sigma_{\mathrm{c}}(\Delta(d, S))=\Pi\left(k^{s_{1}-s_{0}},(k-1)^{s_{2}-s_{1}}, \ldots, 1^{s_{k}-s_{k-1}}\right) .
$$

Proof. Let $P \subset \mathbb{R}^{d}$ be a polytope with vertex set $V$ and let $\ell$ be a linear function. Let $\ell(V)=\left\{a=t_{0}<t_{1}<\cdots<t_{k}=b\right\}$. We write $P_{i}:=P \cap \ell^{-1}\left(t_{i}\right)$ for $0 \leqslant i \leqslant k$. Theorem 1.5 of [4] together with the fact that

$$
P \cap \ell^{-1}\left(\frac{t_{i}+t_{i+1}}{2}\right)=\frac{1}{2}\left(P_{i}+P_{i+1}\right)
$$

for $0 \leqslant i<m$ yields that

$$
(b-a) \Sigma_{\ell}(P)=\frac{1}{2} P_{0}+\sum_{i=1}^{k-1} P_{i}+\frac{1}{2} P_{k} .
$$


If $P=\Delta(d, S)$ and $\ell(\mathbf{x})=\mathrm{c}(\mathbf{x})$, then $P_{i}=\Delta\left(d, s_{i}\right)$ for $0 \leqslant i \leqslant k$. In particular, $P_{0}=\{\mathbf{0}\}$ and $P_{k}=\{\mathbf{1}\}$. Therefore

$$
\frac{1}{2} \mathbf{1}+d \cdot \Sigma_{\mathrm{c}}(\Delta(d, S))=\sum_{i=0}^{k} \Delta\left(d, s_{i}\right) .
$$

Since $\Delta\left(d, s_{i}\right)=\Pi\left(1^{s_{i}}, 0^{d-s_{i}}\right)$ we conclude from Proposition 4 that the above sum is the permutahedron $\Pi(\mathbf{p})$ for

$$
\mathbf{p}=\left(1^{s_{0}}, 0^{d-s_{0}}\right)+\cdots+\left(1^{s_{k}}, 0^{d-s_{k}}\right) .
$$

This finishes the argument.

\section{$5 \quad$ Further questions}

\section{Volumes and Gröbner bases}

Laplace and later Stanley [24] showed that the volume of $\Delta(d, i, i+1)$ is $\frac{A(d, i)}{d !}$ where $A(d, i)$ counts the number of permutations $\sigma$ of $[d]$ with $i$ descents, that is, the number of $1 \leqslant i<d$ such that $\sigma(i)>\sigma(i+1)$; see also [18, 23]. This implies that $d ! \operatorname{vol} \Delta(d,[k, l])$ is the number of permutations of $[d]$ with descent number in $[k, l]=\{k, k+1, \ldots, l\}$ for any $k<l$. It would be very interesting to know if vol $\Delta(d, S)$ has a combinatorial interpretation for all $S$. In light of (2) it would be sufficient to determine vol $\Delta(d, k, l)$ for $l-k>1$.

For $0 \leqslant k<d$, the hypersimplices $\Delta(d, k, k+1) \cong \Delta(d, k+1)$ are alcoved polytopes in the sense of Lam-Postnikov [18] and hence come with a canonical square-free and unimodular triangulation. This is reflected by the fact that the associated toric ideals have quadratic and square-free Gröbner bases with respect to the reverse-lexicographic term order.

For general $k<l$, the polytopes $\Delta(d, k, l)$ are not alcoved anymore. It would be interesting if $\Delta(d, k, l)$ has a unimodular triangulation or square-free Gröbner basis.

\subsection{Extension complexity}

An extension of a polytope $P$ is a polytope $Q$ together with a surjective linear projection $Q \rightarrow P$. The extension complexity $\operatorname{ext}(P)$ of $P$ is the minimal number of facets of an extension of $P$. This is a parameter that is of interest in combinatorial optimization [16]. It was shown in [12] that $\operatorname{ext}(\Delta(d, k, k+1))=2 d$ for $1 \leqslant k \leqslant d-2$.

A realization of the join of two convex polytopes $P, Q \subset \mathbb{R}^{d}$ is given by $P * Q=$ $\operatorname{conv}((P \times \mathbf{0} \times 0) \cup(\mathbf{0} \times Q \times 1))$. If $P$ and $Q$ has $m$ and $n$ facets, respectively, then $P * Q$ has $m+n$ facets. Balas' union bound [1] is the observation that $P * Q \rightarrow P \cup Q$ and hence $\operatorname{ext}(P \cup Q) \leqslant \operatorname{ext}(P)+\operatorname{ext}(Q)$. Iterating the join over the pieces of the decomposition 2 shows the following. 
Proposition 14. If $S \subseteq[0, d]$ is proper, then

$$
\operatorname{ext}(\Delta(d, S)) \leqslant 2 d(|S|-1) .
$$

This is a nontrivial bound as the number of facets of $\Delta(d, S)$ is at least $2+2 d+\sum_{r \notin S}\left(\begin{array}{l}d \\ r\end{array}\right)$. To illustrate, note that the number of facets of the halfcube $H_{d}$ for $d \geqslant 5$ is $2 d+2^{d-1}$ whereas the bounded afforded by Proposition 14 is $\leqslant d^{2}$. Carr and Konjevod [6] gave an extension of $H_{d}$ of size linear in $d$. It would be interesting to know lower bounds on the extension complexity of $\Delta(d, S)$, maybe using the approach via rectangular covering; c.f. $[12]$.

\section{Acknowledgements}

This paper grew out of a project that was part of the course Polytopes, Triangulations, and Applications at Goethe University Frankfurt in spring 2018. We thank Anastasia Karathanasis for her support in the early stages of this project. We also thank Jesús de Loera, Georg Loho, and the anonymous referee for many helpful remarks.

\section{References}

[1] E. BALAs, Disjunctive programming: properties of the convex hull of feasible points, Discrete Appl. Math., 89 (1998), pp. 3-44.

[2] L. J. Billera, M. M. Kapranov, And B. Sturmfels, Cellular strings on polytopes, Proc. Amer. Math. Soc., 122 (1994), pp. 549-555.

[3] L. J. Billera And A. Sarangarajan, The combinatorics of permutation polytopes, in Formal power series and algebraic combinatorics (New Brunswick, NJ, 1994), vol. 24 of DIMACS Ser. Discrete Math. Theoret. Comput. Sci., Amer. Math. Soc., Providence, RI, 1996, pp. 1-23.

[4] L. J. Billera And B. Sturmfels, Fiber polytopes, Ann. of Math. (2), 135 (1992), pp. $527-549$.

[5] K.-H. Borgwardt, The simplex method, vol. 1 of Algorithms and Combinatorics: Study and Research Texts, Springer-Verlag, Berlin, 1987.

[6] R. D. Carr and G. Konjevod, Polyhedral combinatorics, in Tutorials on Emerging Methodologies and Applications in Operations Research, H. J. G, ed., Springer New York, New York, NY, 2005, pp. 2-1-2-46.

[7] H. S. M. Coxeter, Regular polytopes, Dover Publications, Inc., New York, third ed., 1973.

[8] J. A. De Loera, J. Rambau, And F. Santos, Triangulations, vol. 25 of Algorithms and Computation in Mathematics, Springer-Verlag, Berlin, 2010.

[9] R. Edman, P. Jiradilok, G. Liu, and T. McConville, Zonotopes whose cellular strings are all coherent, preprint arXiv:1801.09140, (2018). 
[10] D. Ermel And M. Walter, Parity polytopes and binarization, Discrete Appl. Math., 272 (2020), pp. 24-30.

[11] T. Gosset, On the regular and semi-regular figures in space of $n$ dimensions, Messenger of Mathematics, 29 (1900), pp. 43-48.

[12] F. Grande, A. Padrol, And R. Sanyal, Extension complexity and realization spaces of hypersimplices, Discrete Comput. Geom., 59 (2018), pp. 621-642.

[13] R. M. Green, Homology representations arising from the half cube, Adv. Math., 222 (2009), pp. 216-239.

[14] — Homology representations arising from the half cube, II, J. Combin. Theory Ser. A, 117 (2010), pp. 1037-1048.

[15] M. Grötschel, Cardinality homogeneous set systems, cycles in matroids, and associated polytopes, in The sharpest cut, MPS/SIAM Ser. Optim., SIAM, Philadelphia, PA, 2004, pp. 99-120.

[16] V. KAIBEL, Extended formulations in combinatorial optimization. Optima 85, 2011. 14 pages.

[17] V. Klee and P. Kleinschmidt, Geometry of the Gass-Saaty parametric cost LP algorithm, Discrete Comput. Geom., 5 (1990), pp. 13-26.

[18] T. Lam and A. Postnikov, Alcoved polytopes. I, Discrete Comput. Geom., 38 (2007), pp. 453-478.

[19] D. Maclagan And B. Sturmfels, Introduction to tropical geometry, vol. 161 of Graduate Studies in Mathematics, American Mathematical Society, Providence, RI, 2015.

[20] V. ReINER, The generalized Baues problem, in New perspectives in algebraic combinatorics (Berkeley, CA, 1996-97), vol. 38 of Math. Sci. Res. Inst. Publ., Cambridge Univ. Press, Cambridge, 1999, pp. 293-336.

[21] J. RitTER, The polytopes of cardinality homogeneous set systems, Masterthesis, Freie Universität Berlin, 2016. iii+44 pages.

[22] R. Sanyal, F. Sottile, and B. Sturmfels, Orbitopes, Mathematika, 57 (2011), pp. 275-314.

[23] R. Sanyal And C. Stump, Lipschitz polytopes of posets and permutation statistics, J. Combin. Theory Ser. A, 158 (2018), pp. 605-620.

[24] R. P. Stanley, Eulerian partitions of a unit hypercube, in Higher Combinatorics, M. Aigner, ed., D. Reidel Publishing Co., Dordrecht-Boston, Mass., 1977.

[25] S. Sullivant, Compressed polytopes and statistical disclosure limitation, Tohoku Math. J. (2), 58 (2006), pp. 433-445.

[26] M. YANNAKAKIS, Expressing combinatorial optimization problems by linear programs, J. Comput. System Sci., 43 (1991), pp. 441-466. 